\title{
Persistent eosinophilia and associated organ involvement in Thai patients with systemic sclerosis: Data from the Siriraj scleroderma cohort
}

\author{
Somsak Punjasamanvong (D), Chayawee Muangchan (iD) \\ Department of Internal Medicine, Division of Rheumatology, Faculty of Medicine, Siriraj Hospital, Mahidol University, Bangkok, Thailand
}

\begin{abstract}
Objectives: This study aims to investigate the prevalence of persistent eosinophilia and associated organ complications in Thai patients with systemic sclerosis (SSc).

Patients and methods: This post-hoc study included 107 adult patients ( 23 males, 84 females; mean age: $50.4 \pm 11.6$ years; range, 18 to 79 years) diagnosed with SSc between November 2013 and June 2017. Eosinophilia was defined as an absolute eosinophil count of $>500 / \mu \mathrm{L}$ or a percentage count of $>7 \%$. Eosinophil levels collected at every visit over one year were categorized as persistently high (PH), persistently low (PL), high-to-low $(\mathrm{HL})$, low-to-high $(\mathrm{LH})$, or variable levels $(\mathrm{VL})$. The study compared variables between $\mathrm{PH}$ and non- $\mathrm{PH}(\mathrm{PL}+\mathrm{HL}+\mathrm{LH}+\mathrm{VL})$ groups. The patients with baseline eosinophilia were also identified and compared with the non-eosinophilia group.

Results: The median disease duration was 3.2 years. Of the patients, $79.4 \%$ had diffuse cutaneous SSC and $76.7 \%$ had anti-Scl-70 positivity. A total of $11.2 \%, 66.4 \%, 1.9 \%, 8.4 \%$, and $12.1 \%$ of the patients were categorized into the PH, PL, HL, LH, and VL groups, respectively. Compared to non-PH groups, the PH group had a higher prevalence of anti-centromere antibody (ACA), higher baseline percent predicted total lung capacity, and lower baseline $C$-reactive protein and creatine phosphokinase $(p<0.05$ for all). The ACA positivity (odds ratio [OR]: $18.5 ; 95 \%$ confidence interval [Cl]: 1.64-208.46) was associated with PH. The patients with baseline eosinophilia (17.8\%) had a higher prevalence of non-specific interstitial pneumonia with periodic eosinophilia at the time of diagnosis (100\% vs. $6.5 \%$, p < $0.0001 ;$ OR: 4.667; 95\% Cl: 1.712-12.724).
\end{abstract}

Conclusion: The PH was seldom (11\%) in patients with SSc compared to periodic eosinophilia, which was more prevalent (18\%). It may be related to ACA positivity and better pulmonary outcomes, whereas periodic eosinophilia may involve interstitial lung disease.

Keywords: Eosinophilia complications, eosinophils, post-hoc study, scleroderma, systemic complications.

The hallmark signs of systemic sclerosis (SSc) are widespread microvascular endothelial cell injury, autoimmunity-mediated inflammation, and the development of pathological fibroproliferative lesions. ${ }^{1}$ During the initial phase of the disease, injured endothelial cells and activated platelets upregulate thrombin activity and secrete interleukin (IL)-1, IL-6, and tumor necrosis factor alpha (TNF- $\alpha$ ), which recruit monocytes, macrophages, and fibroblasts into the circulation. ${ }^{1-3}$ Injured endothelial cells, activated platelets, and macrophages further release transforming growth factor beta (TGF- $\beta$ ), platelet-derived growth factor, and connective tissue growth factor, which promote the differentiation of fibroblasts into myofibroblasts. ${ }^{1-3}$ Injured endothelial cells also secrete the alarmin cytokines IL-25, IL-33, and thymic stromal

\section{Received: March 11, 2021 Accepted: April 26, 2021 Published online: October 18, 2021}

Correspondence: Chayawee Muangchan, MD. Department of Internal Medicine, Division of Rheumatology, Faculty of Medicine, Siriraj Hospital, Mahidol University, 10700 Bangkok, Thailand. Tel: +6624197776 e-mail: sichayawee@gmail.com

\section{Citation:}

Punjasamanvong S, Muangchan C. Persistent eosinophilia and associated organ involvement in Thai patients with systemic sclerosis: Data from the Siriraj scleroderma cohort. Arch Rheumatol 2021;36(4):527-537. 
lymphopoietin (TSLP). These cytokines send signals through innate immune cells, including eosinophils, basophils, mast cells, and group 2 innate lymphoid cells, resulting in the upregulation of IL-4, IL-5, IL-9, IL-13, IL-25, and IL-33. These cytokines also promote the differentiation of naïve CD4+ T cells into CD4+ T helper 2 (Th2) cells with resulting type 2 immunity polarization, which contributes to tissue regeneration and fibrosis. ${ }^{4}$ The IL-5 plays an essential role in eosinophil recruitment and activation. ${ }^{5}$ The TSLP, IL-25, and IL-33 promote tissue and blood eosinophilia by inducing IL-5. ${ }^{5}$ The IL-5, leukotriene D4, and granulocyte-macrophage colony-stimulating factor synergistically induce TGF- $\beta$ expression and proteoglycan biosynthesis in eosinophils. ${ }^{6}$ Eosinophils are also partially a source of IL-6, IL-4, and IL-13; as such, they play a role in fibrotic disorders. ${ }^{4,7}$ Via the local generation of eosinophil-derived cationic proteins, endothelial cell cytotoxicity, including procoagulability, may develop, which results in the generation of endovascular fibrosis and intravascular thrombi. Patients with persistent hypereosinophilia may eventually experience endomyocardial fibrosis and arterial and venous thrombotic events. ${ }^{8,9}$

Increases in bronchoalveolar lavage fluid (BALF) eosinophilia are seen in the majority of patients with $\mathrm{SSc}$-associated non-specific interstitial pneumonia (SSc-NSIP), ${ }^{10}$ with more widespread ground-glass opacities on highresolution computed tomography (HRCT) of the chest. ${ }^{11}$ Progression of SSc associated with interstitial lung disease (SSc-ILD) is associated with BALF eosinophilia. ${ }^{12}$ Although BALF eosinophilia is associated with the risk of SSc-ILD progression and mortality, ${ }^{10}$ it is secondary to its link with the extent of SSc-ILD on HRCT of the chest. ${ }^{13}$

An up-to-date cross-sectional study demonstrated a $10 \%$ prevalence of periodic eosinophilia (PerE). ${ }^{14}$ Eosinophilia was significantly prevalent compared to rheumatoid arthritis (RA), primary Sjögren syndrome $(\mathrm{SjS})$, systemic lupus erythematosus (SLE), and mixed connective tissue disease (MCTD). ${ }^{14}$ Periodic eosinophilia was reported to be positively correlated with chest X-ray and HRCT of the chest extent of ILD and modified Rodnan skin score. ${ }^{14}$ However, the likelihood and duration of peripheral blood eosinophilia depend on the physiological or pathological stimuli that cause enhanced eosinophilopoiesis. Without longitudinal data, it is difficult to establish a true cause-and-effect relationship between persistent/PerE and organ complications in SSc. In the present study, we, therefore, aimed to investigate the prevalence of persistent/PerE and associated organ involvement in a longitudinal cohort of Thai patients with SSc.

\section{PATIENTS AND METHODS}

\section{Study design and study population}

This single-center, prospective, observational, post-hoc study was conducted at Siriraj systemic sclerosis clinic, Division of Rheumatology, Department of Internal Medicine, Faculty of Medicine, Siriraj Hospital, Mahidol University, Bangkok, Thailand between November 2013 and June 2017. Adult ( $\geq 18$ years) Thai patients with SSc who met the 1980 American College of Rheumatology (ACR) $)^{15} / 2013$ European League Against Rheumatism (EULAR) ${ }^{16}$ criteria were included to examine the natural course of skin-thickness progression and associated factors. Patients with overlap syndrome who had also met standard classification criteria for RA, SLE, dermatomyositis, polymyositis (PM), and primary $\mathrm{SjS}$ and those with helminthic infections, allergic bronchopulmonary aspergillosis, scabies, drug hypersensitivity, atopic disease, hypereosinophilic syndrome, leukemia, lymphomas, and solid cancers in historical and clinical backgrounds were excluded. In addition, the SSc cohort excluded MCTD and SLE patients with anti-U1RNP positivity, as these patients had several features similar to those of $\mathrm{SSc}$, but had specific autoantibodies, mechanisms of pathogenesis, and unique clinical progressions quite different from SSc. This study also excluded patients who received systemic corticosteroid doses equivalent to $\geq 0.5 \mathrm{mg} / \mathrm{kg} /$ day of prednisolone since the baseline visit. Eligible patients were followed for at least one year and had results collected from at least three complete blood count investigations. Of a total of 164 SSc patients assessed for eligibility, 23 were lost to follow-up, four died, and two were referred to another center, and 135 had complete one-year follow-up. Twenty-eight of these 135 patients 
had overlap syndromes of RA (20/28), SLE (3/28), PM (2/28), RA+SLE (2/28), and SLE+PM $(1 / 28)$. Finally, a total of 107 adult SSc patients (23 males, 84 females; mean age: $50.4 \pm 11.6$ years; range, 18 to 79 years) were included in the study. A written informed consent was obtained from each patient. The study protocol was approved by the Siriraj Hospital Institutional Review Board (No: Si229/2017). The study was conducted in accordance with the principles of the Declaration of Helsinki.

\section{Clinical and laboratory parameters}

The definitions of the clinical and laboratory parameters used in this study were previously described. ${ }^{17}$ The World Health Organization defined eosinophilia by the presence of an increased number of peripheral blood eosinophils exceeding the upper limit of normal, 3 to $-5 \%$ white blood cell count, or an absolute white blood cell count of 350 to $500 / \mu \mathrm{L} .{ }^{18}$ Reference intervals for absolute eosinophil count (AEC) (and percent eosinophil count [PEC]) in healthy Thai adults were 100 to $800 / \mu \mathrm{L}(0.8$ to $9.2 \%)$ in males and 30 to $500 / \mu \mathrm{L}$ (0.4 to $7.5 \%$ ) in females, respectively. ${ }^{19}$ Thus, in this study an AEC of $>500 / \mu \mathrm{L}$ or a PEC of $>7 \%$ was defined as eosinophilia. This study collected AEC and PEC at every visit and, then, plotted the values to define pattern changes over one year. Indeed, the numbers of visits over one year by each patient were unequal. Visit numbers varied from 6 to 12 visits and, therefore, if a group of patients had six visits, the corresponding linear plot would be shorter than for a group of patients who had 12 visits. There was no standard classification that defined pattern changes of eosinophilia. Based on the definition of hypereosinophilia as a blood AEC of $\geq 1,500 / \mu \mathrm{L}$ obtained on at least two separate occasions (interval $\geq 1$ month) ${ }^{20}$ and the fact that eosinophilia could be transient, episodic, or persistent, patients were arbitrarily categorized into one of the following five groups based on the corresponding linear plots over one year (longitudinal perspective): persistently high (PH) group, persistently low (PL) group, high-to-low (HL) group, low-to-high (LH) group, or variable levels (VL) group. These data were, then, used to further categorize the $\mathrm{PH}$ group into the eosinophilia group and the $\mathrm{PL}+\mathrm{HL}+\mathrm{LH}+\mathrm{VL}$ (non-PH) groups into the non-eosinophilia group. At the baseline visit, the patients were also categorized into baseline eosinophilia and non-eosinophilia groups (cross-sectional perspective). In this case, patients who had baseline eosinophilia were defined as having PerE.

\section{Statistical analysis}

Ando et al. ${ }^{14}$ reported a $71 \%$ and $37 \%$ prevalence of ILD in patients with and without eosinophilia, respectively. Using the predefined definition of pattern changes of eosinophilia, we estimated the prevalence of ILD to be $71 \%$ in the $\mathrm{PH}$ group and $37 \%$ in all of the other groups combined (non-PH group). Moreover, the ratio of the non-eosinophilia group to the $\mathrm{PH}$ group was 2:1. A sample size of 135 patients was calculated, ensuring a 95\% confidence interval $(95 \% \mathrm{CI})$ $(Z=1.96)$, a level of significance of 0.05 , and power of the test of $80 \%$.

Statistical analysis was performed using the PASW version 18.0 software (SPSS Inc., Chicago, IL, USA). Continous variables were expressed in mean \pm standard deviation (SD) for normally distributed variables and in median $\left(25^{\text {th }}-75^{\text {th }}\right.$ percentile, interquartile range [IQR]) for non-normally distributed variables. Categorical variables were expressed in number and frequency. For comparisons between two groups, the Student t-test or the Mann-Whitney $\mathrm{U}$ test was used for continuous variables, while the chi-square test or Fisher exact test was used for categorical variables. For the relationship between the PH group and variables of interest, the Spearman correlation coefficient statistic was used for all variables. Variables with a $p$ value of $<0.2$ were included in multivariate analysis. Univariate and multivariate variables of interest were evaluated using binary logistic regression analysis (Forward: LR method), with the results presented in odds ratio (OR) and 95\% CI. A two-tailed $p$ value of $<0.05$ was considered statistically significant.

\section{RESULTS}

Of the patients, $79.4 \%$ had diffuse cutaneous SSc (dcSSc), 76.6\% had anti-Scl-70 positivity, and the median (IQR) disease duration from onset of non-Raynaud symptoms to the first visit was 3.2 (IQR: 1.1 to 6.8 ) years (Table 1). Among the entire 


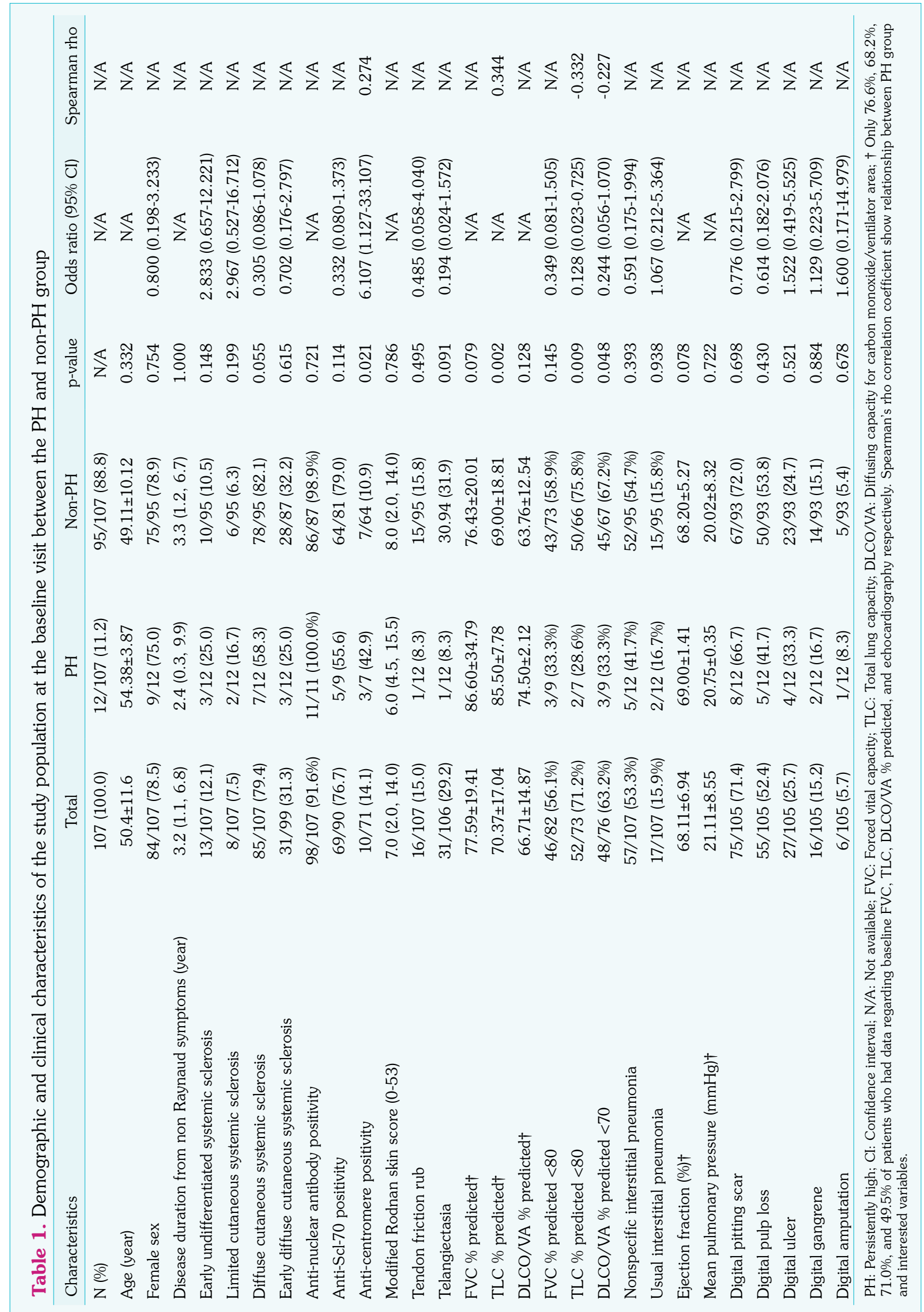



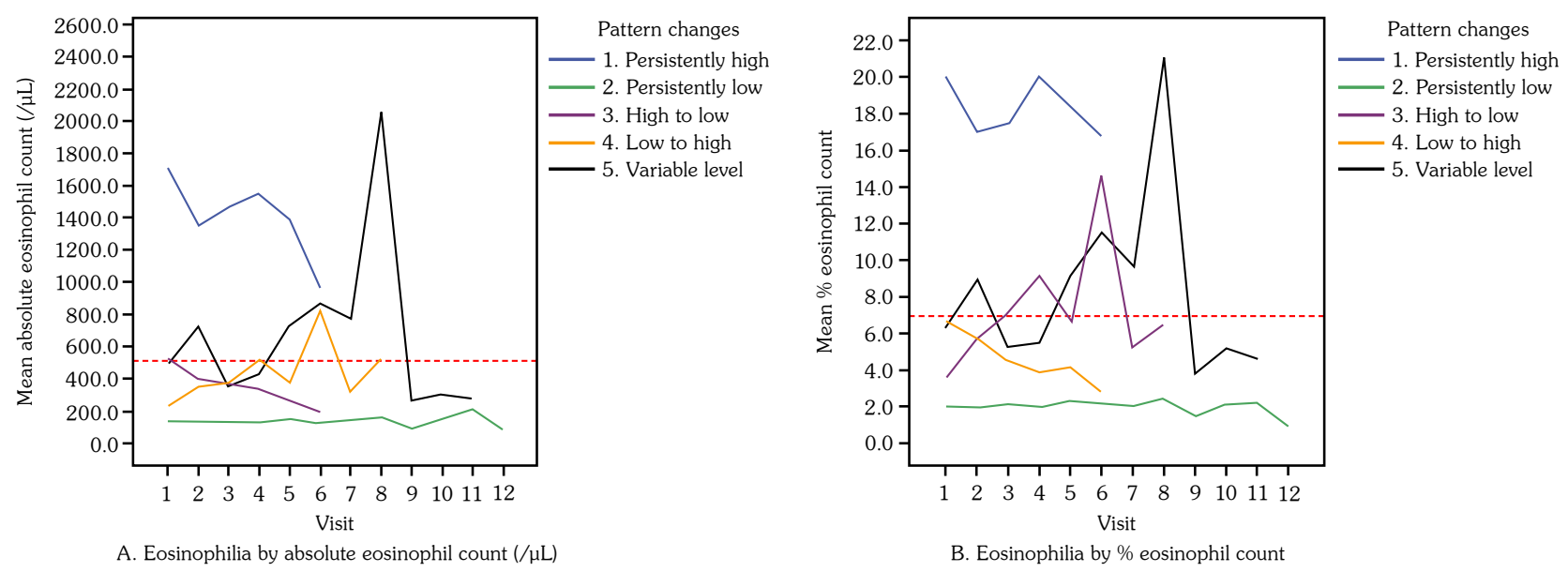

Figure 1. A linear plot of pattern changes of eosinophilia over one year. The linear plots represented the corresponding mean eosinophil counts in Y-axis and visit number in X-axis. The visit numbers of each patient were unequal. Visits numbers varied from 6 to 12 visits and, therefore, if a group of patients had six visits, the corresponding linear plot would shorter than the group of patients who had 12 visits.

cohort, 19 of 107 (17.8\%) patients had baseline or PerE, whereas 12 of 107 (11.2\%) had persistent eosinophilia (PH group), and the PL group had the most considerable proportion of patients (66.4\%). The HL, LH, and VL groups accounted for $1.9 \%, 8.4 \%$, and $12.1 \%$ of the patients, respectively. In the $\mathrm{PH}$ group, the AEC and $\mathrm{PEC}$ ranged from 480.34 to $4,092.48 / \mu \mathrm{L}$ and from $5.8 \%$ to $51.1 \%$, respectively. The $\mathrm{PH}$ group was designed with an AEC of $>500 / \mu \mathrm{L}$ or a PEC $>7 \%$. Eight of 12 patients fulfilled both criteria, whereas four patients fulfilled either of them. Two of these had an AEC of $488.58 / \mu \mathrm{L}$ and $480.34 / \mu \mathrm{L}$ at one visit, whereas the other two had a PEC of $6.3 \%$ and $5.8 \%$ at one visit. A linear plot of each group over one year is shown in Figure 1.

No significant difference was observed between $\mathrm{PH}$ and non-PH groups with regard to age, sex, and disease duration. However, there was a non-significant trend toward a decreased prevalence of $\mathrm{dcSSc}$ subset in the $\mathrm{PH}$ group (58.3\% vs. $82.1 \%$, respectively; $\mathrm{p}=0.055)$. The $\mathrm{PH}$ group had significantly more anti-centromere antibody (ACA) positivity than the non-PH group (42.9\% vs. $10.9 \%$, respectively; $p=0.021$ ), which correlated positively $(\mathrm{r}=0.274 ; \mathrm{p}=0.021)$. The $\mathrm{PH}$ group had a better mean baseline percent predicted (\%predicted) total lung capacity (TLC) $(85.50 \pm 7.78$ vs. $69.00 \pm 18.81$, respectively; $\mathrm{p}=0.002$ ), whereas there was a positive correlation $(r=0.344 ; p=0.004)$. The rates of baseline \%predicted TLC $<80$ and \%predicted diffusing capacity for carbon monoxide/ventilator area (DLCO/VA) $<70$ were less in the $\mathrm{PH}$ group ( $28.6 \%$ vs. $75.8 \%$, respectively; $p=0.009$ and $33.3 \%$ vs. $67.2 \%$, respectively; $p=0.048$ ). The poor \%predicted TLC and \%predicted DLCO/VA also correlated negatively with the $\mathrm{PH}$ group $(\mathrm{r}=-0.332, \mathrm{p}=0.005$ and $\mathrm{r}=-0.227, \mathrm{p}=0.049$, respectively) (Table 1).

The PH group had a significantly lower median baseline C-reactive protein (CRP) (1.5 [IQR: 0.5 to 1.9 ] vs. 3.0 [IQR: 1.0 to 7.9 ]; $\mathrm{p}=0.031$ ) and baseline creatine phosphokinase (CPK) (89.5 [IQR: 71.8 to 109.3] vs. 116.0 [IQR: 79.5 to 169.5$] ; p=0.041)$ compared to the non-PH group. The PH group also correlated negatively with baseline CRP and baseline CPK $(\mathrm{r}=-0.217$, $p=0.028$ and $r=-0.217, p=0.028$, respectively) (Table 2). Regarding disease progression at one year compared between the $\mathrm{PH}$ and non-PH groups, the rates of one-year \%predicted TLC $<80$ were also less in the $\mathrm{PH}$ group $(0.0 \%$ vs. $72.7 \%$, respectively; $\mathrm{p}=0.011$ ). However, the patients who underwent follow-up pulmonary function tests were only small in number (Table 3).

After adjustment for age, female sex, disease duration from onset of non-Raynaud phenomenon symptoms to the first visit, dcSSc subset (yes/no), anti-Scl-70 positivity (yes/no), ACA positivity 


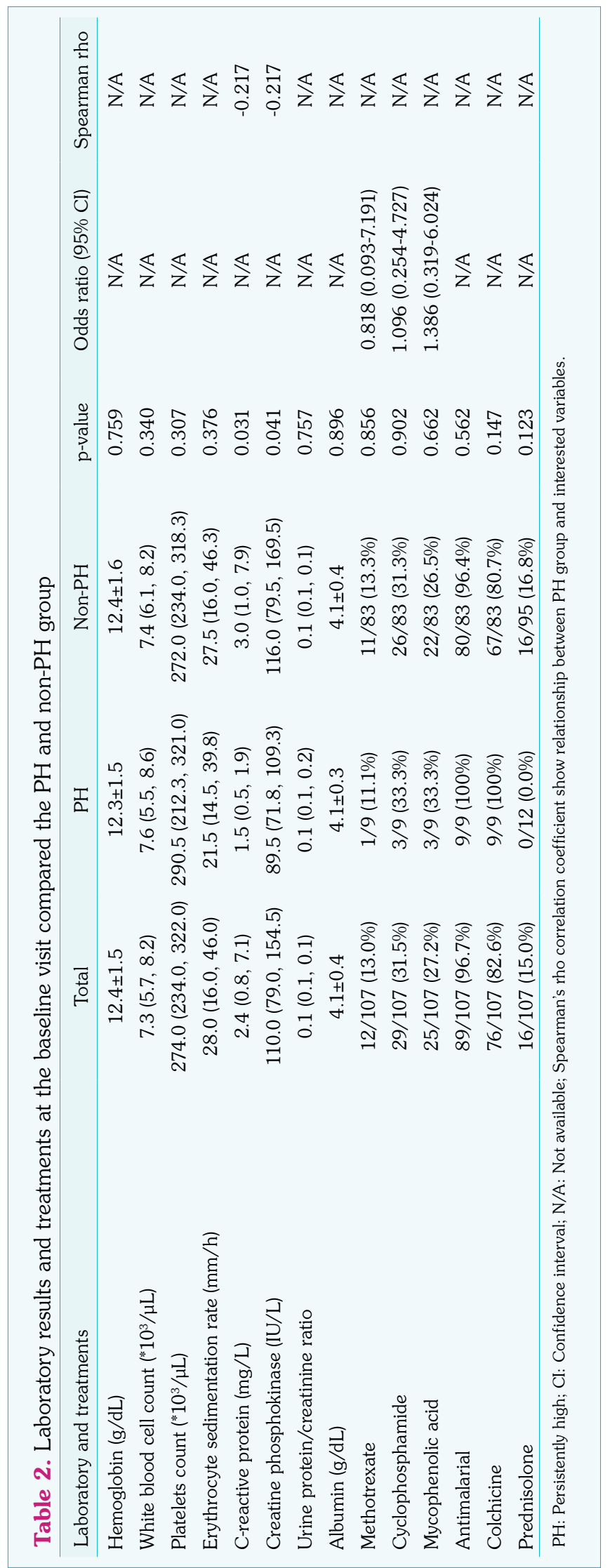


Table 3. Clinical and laboratory progression at one-year compared between the PH and non-PH group

\begin{tabular}{lccccc}
\hline $\begin{array}{l}\text { Clinical and laboratory progression } \\
\text { at one-year }\end{array}$ & Total & PH & Non-PH & p-value & Odds ratio (95\% CI) \\
\hline Mean \pm SD change mRSS (N) & $-6.3 \pm 6.5(107)$ & $-8.0 \pm 8.7(12)$ & $-6.0 \pm 6.3(95)$ & 0.331 & N/A \\
1-year mRSS <10 & $93 / 107(86.9 \%)$ & $11 / 12(91.7 \%)$ & $82 / 95(86.3 \%)$ & 0.605 & $1.744(0.207-14.661)$ \\
1-year FVC \%predicted <80† & $24 / 38(63.2 \%)$ & $2 / 3(66.7 \%)$ & $22 / 35(62.9 \%)$ & 0.896 & $1.182(0.097-14.347)$ \\
1-year TLC \%predicted <80† & $24 / 36(66.7 \%)$ & $0 / 3(0.0 \%)$ & $24 / 33(72.7 \%)$ & 0.011 & N/A \\
1-year DLCO/VA \%predicted $<70 \dagger$ & $20 / 36(55.6 \%)$ & $1 / 3(33.3 \%)$ & $19 / 33(57.6 \%)$ & 0.418 & $0.368(0.030-4.478)$ \\
1-year ESR <20 mm/h. & $35 / 105(33.3 \%)$ & $5 / 12(41.7 \%)$ & $30 / 93(32.3 \%)$ & 0.515 & $1.500(0.440-5.118)$ \\
1-year CRP (mg/L) & $1.9(0.6,5.0)$ & $1.0(0.6,2.2)$ & $2.2(0.6,5.1)$ & 0.124 & $\mathrm{~N} / \mathrm{A}$ \\
1-year CRP <1 mg/L & $34 / 102(33.3 \%)$ & $6 / 12(50.0 \%)$ & $28 / 90(31.1 \%)$ & 0.192 & $2.214(0.656-7.473)$ \\
1-year CPK <190 IU/L & $89 / 105(84.8 \%)$ & $12 / 12(100 \%)$ & $77 / 93(82.8 \%)$ & 0.119 & $\mathrm{~N} / \mathrm{A}$ \\
New digital pitting scar & $54 / 107(50.5 \%)$ & $5 / 12(41.7 \%)$ & $49 / 95(51.6 \%)$ & 0.518 & $0.671(0.199-2.263)$ \\
New digital pulp loss & $48 / 107(44.9 \%)$ & $7 / 12(58.3 \%)$ & $41 / 95(43.2 \%)$ & 0.319 & $1.844(0.546-6.229)$ \\
New digital ulcer & $16 / 107(15.0 \%)$ & $1 / 12(8.3 \%)$ & $15 / 95(15.8 \%)$ & 0.495 & $0.485(0.058-4.040)$ \\
New digital gangrene & $2 / 107(1.9 \%)$ & $0 / 12(0.0 \%)$ & $2 / 95(2.1 \%)$ & 0.612 & N/A \\
New digital amputation & $7 / 107(6.5 \%)$ & $2 / 12(16.7 \%)$ & $5 / 95(5.3 \%)$ & 0.132 & $3.600(0.616-21.033)$ \\
New any digital ischemic complications & $71 / 107(66.4 \%)$ & $7 / 12(58.3 \%)$ & $64 / 95(67.4 \%)$ & 0.533 & $0.678(0.199-2.309)$ \\
\hline
\end{tabular}

PH: Persistently high; CI: Confidence interval; mRSS: Modified Rodnan skin score; N/A: Not available; FVC: Forced vital capacity; TLC: Total lung capacity; DLCO/VA: Diffusing capacity for carbon monoxide/ventilator area; ESR: Erythrocyte sedimentation rate; CRP: C-reactive protein; CPK: Creatine phosphokinase; † Only 35.5\%, 33.6\%, and 33.6\% of 107 patients who had data regarding 1-year FVC, TLC, and DLCO/VA \%predicted, respectively.

Table 4. Prevalence of NSIP between baseline eosinophilia and non-eosinophilia group

\begin{tabular}{lccccc}
\hline & Total & Eosinophilia & $\begin{array}{c}\text { Non- } \\
\text { eosinophilia }\end{array}$ & p-value & Odds ratio (95\% CI) \\
\hline N (\%) & $107(100 \%)$ & $19 / 107(17.8 \%)$ & $88 / 107(82.2 \%)$ & N/A & N/A \\
All NSIP & $57 / 107(53.3 \%)$ & $11 / 19(57.9 \%)$ & $46 / 88(52.3 \%)$ & 0.656 & $1.255(0.461,3.420)$ \\
NSIP diagnosed 2-year before or after visit 1 & $42 / 57(73.7 \%)$ & $9 / 11(81.8 \%)$ & $33 / 46(71.7 \%)$ & 0.495 & $1.773(0.337,9.334)$ \\
NSIP with eosinophilia occurred in time of & $14 / 57(24.6 \%)$ & $11 / 11(100 \%)$ & $3 / 46(6.5 \%)$ & $<0.0001$ & $4.667(1.712,12.724)$ \\
diagnosis & & & & & \\
\hline
\end{tabular}

NSIP: Non-specific interstitial pneumonia; CI: Confidence interval; N/A: Not available; † Univariable odds ratios between baseline eosinophilia and noneosinophilia group.

(yes/no), baseline \%predicted TLC $<80$ (yes/no), baseline CRP, and baseline CPK, ACA positivity (OR: $18.5 ;$ 95\% CI: $1.642-208.462 ; \mathrm{p}=0.018$ ) was independently associated with the PH group.

From the cross-sectional perspective, patients with baseline or PerE group showed no significant difference regarding the prevalence of NSIP. Among the entire cohort, 57 of 107 (53.3\%) patients had baseline SSc-NSIP. Most of these patients $(73.7 \%)$ had SSc-NSIP early on in the disease course (two years before or after the first visit). One-fourth (24.6\%) had eosinophilia that occurred a few months before or after the diagnosis of SSc-NSIP. Within this subset of patients, the PerE group had significantly more SSc-NSIP with eosinophilia than the non-PerE group (100\% vs. 6.5\%, respectively; $p<0.0001$; OR: 4.667; 95\% CI: 1.712-12.724) (Table 4).

\section{DISCUSSION}

Endothelial cell damage occurs early in the course of SSc pathogenesis. ${ }^{21}$ The IL-33, which is a member of the IL-1 family, is immediately released after endothelial cell damage and has been reported to significantly increase in early 
SSc, which promotes $\mathrm{TH} 2$ polarization and IL-13-dependent fibrosis. ${ }^{22-24}$ The TSLP, IL-25, or IL-17E and IL-33 from damaged endothelial cells promote eosinophilia by inducing IL- 5 , which is a TH2-type cytokine, from CD4+ TH2 cells; ${ }^{5}$ hence, secondary eosinophilia occurs in response to the increased production of IL- $5,{ }^{25}$ which may play a role in the inflammatory and fibrotic process in SSc development. However, there is no evidence regarding the significance of IL-5 in SSc, although PerE in SSc has been reported in cross-sectional studies. ${ }^{14,26}$ Considering these data, this study set forth to determine the significance of eosinophils in SSc patients.

Wuttge et al. ${ }^{27}$ reported characteristics of peripheral blood eosinophils in SSc patients. Eosinophil counts in peripheral blood increased with disease duration from onset of symptoms of non-Raynaud phenomenon $(\mathrm{r}=0.55, \mathrm{p}=0.001)$, while short disease duration ( $<2$ years) produced eosinophil counts similar to those in healthy controls. The SSc patients with long disease duration ( $\geq 2$ years) had higher eosinophil counts. The CD81-expressed eosinophils were lower in SSc patients compared to controls, irrespective of disease duration, while CD48-expressed eosinophils increased in SSc patients with a short disease duration and were associated with alveolitis as defined by increased concentration of alveolar nitric oxide. This finding could infer a change in eosinophil phenotype during disease progression in SSc.

The prevalence of periodic (i.e., presence of eosinophilia at baseline visit) and persistent eosinophilia over a year in this retrospective study of a prospective cohort of SSc patients was $17.8 \%$ and $11.2 \%$, respectively. Most patients (66.4\%) in the present study had PL eosinophilia throughout the year. Ando et al., ${ }^{14}$ using a percentage count of $>7 \%$, reported a $10 \%$ prevalence of PerE in 70 SSc patients with 4.9 years of disease duration. Foocharoen et al. ${ }^{26}$ using an absolute count of $>500 / \mu \mathrm{L}$, reported a $21.9 \%$ prevalence of PerE in 164 SSc patients with 6.5 years of disease duration. The latter study also found that disease duration was associated with eosinophilia (OR: 1.16, 95\% CI: 1.03-1.30). In the present study, 36.5\% and $47.9 \%$ of the entire cohort had disease duration of less than two and less than three years, respectively; the overall median disease duration was only 3.2 years. Moreover, PerE was less frequent than in patients in the Foocharoen et al.'s ${ }^{26}$ study, due to shorter disease duration, although it was more prevalent than was reported by Ando et al. ${ }^{14}$ The more frequent occurrence of PerE in the present study can be explained by the higher prevalence of SSc-NSIP (53.3\%) in comparison with that reported by Ando et al. ${ }^{14}$ (34\%).

Furthermore, SSc can involve systemic inflammation at low grade as defined by CRP levels, particularly in early disease, which correlates with reduced pulmonary function. ${ }^{28}$ Systemic sclerosis-NSIP appears to complicate $\mathrm{SSc}$ most frequently early on in the disease course. ${ }^{13}$ One-quarter of SSc-NSIP patients in the present study had eosinophilia that occurred around the time of diagnosis. Also, SSc-NSIP with eosinophilia was found to be more frequent in the PerE group. This finding is consistent with previous results reported by Ando et al. ${ }^{14}$ and Wuttge et al. ${ }^{27}$ The PerE group may represent the inflammatory process that activates peripheral blood eosinophils, facilitating their recruitment to the alveoli.

In the current study, persistent eosinophilia was associated with a different phenomenon. The SSc patients with persistent eosinophilia had a trend toward a lesser degree of severity. They were less likely to belong to the dcSSc subset and had greater ACA positivity than those with non-persistent eosinophilia. They had also a lower-level systemic inflammatory process at baseline defined by CRP and CPK. Finally, they had a better baseline and one-year pulmonary outcome. ${ }^{28}$ However, the present study could not demonstrate longer disease duration in patients with persistent eosinophilia compared to those with non-persistent eosinophilia due to the relatively small sample size of the total cohort.

The ACA positivity was an independent protective factor against the development of dcSSc and fibrotic ILD. ${ }^{29,30}$ Similarly, the absence of ACA was an independent predictor of fibrotic ILD. ${ }^{31}$ There is currently no evidence supporting an association between ACA positivity and persistent eosinophilia. Higher CRP levels were associated with the absence of ACA and correlated with concomitant lung, cutaneous, and 
musculoskeletal morbidity. ${ }^{28,32}$ Higher baseline CRP levels independently predicted a decline in percent predicted forced vital capacity (FVC). ${ }^{32}$ HyperCKemia from bland myopathy was also associated with poor \%predicted FVC. ${ }^{33,34}$ Although persistent eosinophilia in SSc patients correlated with a lesser degree of severity, it might be beyond the scope of the present study to conclude that persistent eosinophilia did not cause any other long-term ( $>1$ year) organ complications. However, there was a nonsignificant trend toward an increased prevalence of new digital amputation in the PH group $(16.7 \%$ vs. $5.3 \%$, respectively; $p=0.132$; OR: $3.6 ; 95 \%$ CI: 0.616-21.033).

At study baseline, no patients received prednisolone doses higher than $30 \mathrm{mg} /$ day or $0.5 \mathrm{mg} / \mathrm{kg} /$ day. Moreover, no patients in the $\mathrm{PH}$ group received prednisolone during the one-year follow-up. However, 16 SSc patients of $95(16.8 \%)$ in the non-PH group received prednisolone during the year, 15 of whom had doses of $\leq 15 \mathrm{mg} /$ day. Nevertheless, low-dose prednisolone seemed to have an antagonistic effect on peripheral blood eosinophil counts. A gradual tapering of prednisolone dosage contributed to increased levels of peripheral blood eosinophils. ${ }^{35}$ One patient was possibly misclassified into the LH group instead of the $\mathrm{PH}$ group, due to low levels of peripheral blood eosinophils. This patient received prednisolone at a dose of $5 \mathrm{mg} /$ day which was, then, tapered to $2.5 \mathrm{mg} /$ day during the first two visits; thereafter, eosinophil counts increased to the levels of persistent eosinophilia.

The main limitation of this study is the absence of serological evidence or other diagnostic tests to rule out nematodes, filariasis, flukes, and protozoan infections. Furthermore, pruritusspecific quantitative data were not available. However, severe allergic rhinitis, asthma, extensive atopic/contact dermatitis, chronic sinusitis, eosinophilic granulomatosis with polyangiitis, and hypereosinophilic syndrome were not documented in our retrospective review. The present study could not investigate the eosinophilia of healthy control individuals and patients with other connective tissue diseases such as RA, SLE, dermatomyositis, PM, and MCTD. Moreover, the study had limited ability in deducing causal associations given its observational nature, mostly by analyzing baseline characteristics. The PH group, itself, was very heterogeneous and small in number, indicating that we could not discern any clear clinical difference between the patients with very high and relatively low persistent eosinophilia. It was not known precisely how many dosages and magnitude of systemic corticosteroids affect peripheral blood eosinophil levels. Ortega et al. ${ }^{36}$ found an average dose of $35 \mathrm{mg} /$ day of prednisolone decreased peripheral blood eosinophil counts by $30 \%$. We inferred that based on the average weight of Thai patients, $\geq 0.5 \mathrm{mg} / \mathrm{kg} /$ day of prednisolone might have significantly affected peripheral blood eosinophil levels. However, the lower dosages seemed to have an antagonistic effect on peripheral blood eosinophil counts, as one patient was possibly misclassified into the LH group instead of the $\mathrm{PH}$ group. Overall, the intended sample size of 135 could not be attained; however, SSc is a rare disease, and data from 107 patients constitute a substantial total. Further studies conducted over a more extensive period are needed to investigate whether persistent eosinophilia in SSc may be associated with other organ complications.

In conclusion, persistent eosinophilia was infrequent in Thai patients with SSc in contrast to PerE, which was considerably more prevalent. However, nearly $70 \%$ of SSc patients had absolute and percent eosinophil counts within their normal limits. Based on these findings, persistent eosinophilia may be an epiphenomenon of or a surrogate marker for a distinct SSc subset that indicates better pulmonary outcome rather than causally influencing the pathological process of SSc disease; however, the effect of long-standing eosinophilia in SSc patients needs to be further elucidated.

\section{Acknowledgments}

We gratefully acknowledge the patients who participated in this study, Ms. Khemajira Karaketklang (Research and Academic Service Unit, Department of Internal Medicine, Faculty of Medicine Siriraj Hospital, Mahidol University, Bangkok, Thailand) for assistance with statistical analysis, and Asst. Prof. Kevin P. Jones (Siriraj Medical Research Center, Faculty of Medicine Siriraj Hospital, Mahidol University, Bangkok, Thailand) for medical research manuscript editing. We thank Hugh McGonigle, from Edanz Group for editing a draft of the manuscript. 


\section{Declaration of conflicting interests}

The authors declared no conflicts of interest with respect to the authorship and/or publication of this article.

\section{Funding}

The authors received no financial support for the research and/or authorship of this article.

\section{REFERENCES}

1. Feghali-Bostwick C, Varga J. Introduction: The etiopathogenesis of systemic sclerosis-an integrated overview. In: Varga J, Denton CP, Wigley FM, Allanore Y, Kuwana M, editors. Scleroderma: from pathogenesis to comprehensive management. 2nd ed. New York: Springer; 2017. p. 133-9.

2. Pattanaik D, Brown M, Postlethwaite BC, Postlethwaite AE. Pathogenesis of systemic sclerosis. Front Immunol 2015;6:272.

3. Akter T, Silver RM, Bogatkevich GS. Recent advances in understanding the pathogenesis of sclerodermainterstitial lung disease. Curr Rheumatol Rep 2014;16:411.

4. Gieseck RL 3rd, Wilson MS, Wynn TA. Type 2 immunity in tissue repair and fibrosis. Nat Rev Immunol 2018;18:62-76.

5. Rosenberg HF, Dyer KD, Foster PS. Eosinophils: Changing perspectives in health and disease. Nat Rev Immunol 2013;13:9-22.

6. Kato Y, Fujisawa T, Nishimori H, Katsumata H, Atsuta $\mathrm{J}$, Iguchi $\mathrm{K}$, et al. Leukotriene $\mathrm{D} 4$ induces production of transforming growth factor-beta1 by eosinophils. Int Arch Allergy Immunol 2005;137 Suppl 1:17-20.

7. Gu YS, Kong J, Cheema GS, Keen CL, Wick G, Gershwin ME. The immunobiology of systemic sclerosis. Semin Arthritis Rheum 2008;38:132-60.

8. Roufosse F. L4. Eosinophils: How they contribute to endothelial damage and dysfunction. Presse Med 2013;42:503-7.

9. Liapis H, Ho AK, Brown D, Mindel G, Gleich G. Thrombotic microangiopathy associated with the hypereosinophilic syndrome. Kidney Int 2005;67:1806-11.

10. Bouros D, Wells AU, Nicholson AG, Colby TV, Polychronopoulos V, Pantelidis P, et al. Histopathologic subsets of fibrosing alveolitis in patients with systemic sclerosis and their relationship to outcome. Am J Respir Crit Care Med 2002;165:1581-6.

11. Hesselstrand R, Wildt M, Bozovic G, AnderssonSjöland A, Andréasson K, Scheja A, et al. Biomarkers from bronchoalveolar lavage fluid in systemic sclerosis patients with interstitial lung disease relate to severity of lung fibrosis. Respir Med 2013;107:1079-86.
12. De Santis M, Bosello SL, Peluso G, Pinnelli M, Alivernini S, Zizzo G, et al. Bronchoalveolar lavage fluid and progression of scleroderma interstitial lung disease. Clin Respir J 2012;6:9-17.

13. Mackintosh JA, Stainer A, Barnett JL, Renzoni EA. Systemic sclerosis associated interstitial lung disease: A comprehensive overview. Semin Respir Crit Care Med 2019;40:208-26.

14. Ando K, Nakashita T, Kaneko N, Takahashi K, Motojima S. Associations between peripheral blood eosinophil counts in patients with systemic sclerosis and disease severity. Springerplus 2016;5:1401.

15. Preliminary criteria for the classification of systemic sclerosis (scleroderma). Subcommittee for scleroderma criteria of the American Rheumatism Association Diagnostic and Therapeutic Criteria Committee. Arthritis Rheum 1980;23:581-90.

16. van den Hoogen F, Khanna D, Fransen J, Johnson SR, Baron M, Tyndall A, et al. 2013 classification criteria for systemic sclerosis: An American college of rheumatology/ European league against rheumatism collaborative initiative. Ann Rheum Dis 2013;72:1747-55.

17. Wannarong $T$, Muangchan $C$. High burden of skin sclerosis is associated with severe organ involvement in patients with systemic sclerosis and systemic sclerosis overlap syndrome. Rheumatol Int 2018;38:2279-88.

18. Shomali W, Gotlib J. World Health Organizationdefined eosinophilic disorders: 2019 update on diagnosis, risk stratification, and management. Am J Hematol 2019;94:1149-67.

19. Wongkrajang $P$, Chinswangwatanakul W, Mokkhamakkun C, Chuangsuwanich N, Wesarachkitti $\mathrm{B}$, Thaowto B, et al. Establishment of new complete blood count reference values for healthy Thai adults. Int $J$ Lab Hematol 2018;40:478-83.

20. Valent P, Klion AD, Horny HP, Roufosse F, Gotlib J, Weller PF, et al. Contemporary consensus proposal on criteria and classification of eosinophilic disorders and related syndromes. J Allergy Clin Immunol 2012;130:607-12.e9.

21. Gualtierotti R, Ingegnoli F, Griffini S, Grovetti E, Borghi MO, Bucciarelli P, et al. Detection of early endothelial damage in patients with Raynaud's phenomenon. Microvasc Res 2017;113:22-8.

22. Vettori S, Cuomo G, Iudici M, D'Abrosca V, Giacco V, Barra G, et al. Early systemic sclerosis: Serum profiling of factors involved in endothelial, T-cell, and fibroblast interplay is marked by elevated interleukin-33 levels. $J$ Clin Immunol 2014;34:663-8.

23. Manetti M, Guiducci S, Ceccarelli C, Romano E, Bellando-Randone S, Conforti ML, et al. Increased circulating levels of interleukin 33 in systemic sclerosis correlate with early disease stage and microvascular involvement. Ann Rheum Dis 2011;70:1876-8.

24. Rankin AL, Mumm JB, Murphy E, Turner S, Yu N, McClanahan TK, et al. IL-33 induces IL-13-dependent cutaneous fibrosis. J Immunol 2010;184:1526-35. 
25. Roufosse F, Weller PF. Practical approach to the patient with hypereosinophilia. J Allergy Clin Immunol 2010;126:39-44.

26. Foocharoen C, Kongkulkiat P, Mahakkanukrauh A, Intapan PM, Maleewong W, Jumnainsong $A$, et al. SAT0299 prevalence of peripheral eosinophilia and clinical associations in Thai systemic sclerosis patients. Annals of the Rheumatic Diseases 2019;78:1226-7.

27. Wuttge DM, Andreasson A, Tufvesson E, Johansson $\AA$ A , Scheja A, Hellmark T, et al. CD81 and CD48 show different expression on blood eosinophils in systemic sclerosis: New markers for disease and pulmonary inflammation? Scand J Rheumatol 2016;45:107-13.

28. Muangchan C, Harding S, Khimdas S, Bonner A; Canadian Scleroderma Research group, Baron M, Pope J. Association of C-reactive protein with high disease activity in systemic sclerosis: Results from the Canadian Scleroderma Research Group. Arthritis Care Res (Hoboken) 2012;64:1405-14.

29. Iniesta Arandia N, Simeón-Aznar CP, Guillén Del Castillo A, Colunga Argüelles D, Rubio-Rivas M, Trapiella Martínez L, et al. Influence of antibody profile in clinical features and prognosis in a cohort of Spanish patients with systemic sclerosis. Clin Exp Rheumatol 2017;35 Suppl 106:98-105.

30. Wangkaew S, Euathrongchit J, Wattanawittawas $\mathrm{P}$, Kasitanon $\mathrm{N}$, Louthrenoo $\mathrm{W}$. Incidence and predictors of interstitial lung disease (ILD) in Thai patients with early systemic sclerosis: Inception cohort study. Mod Rheumatol 2016;26:588-93.
31. Simeón-Aznar CP, Fonollosa-Plá V, Tolosa-Vilella C, Espinosa-Garriga G, Ramos-Casals M, Campillo-Grau $\mathrm{M}$, et al. Registry of the Spanish network for systemic sclerosis: Clinical pattern according to cutaneous subsets and immunological status. Semin Arthritis Rheum 2012;41:789-800.

32. Liu X, Mayes MD, Pedroza C, Draeger HT, Gonzalez $\mathrm{EB}$, Harper BE, et al. Does C-reactive protein predict the long-term progression of interstitial lung disease and survival in patients with early systemic sclerosis? Arthritis Care Res (Hoboken) 2013;65:1375-80.

33. Jung M, Bonner A, Hudson M, Baron M, Pope JE; Canadian Scleroderma Research Group (CSRG). Myopathy is a poor prognostic feature in systemic sclerosis: Results from the Canadian Scleroderma Research Group (CSRG) cohort. Scand J Rheumatol 2014;43:217-20.

34. Paik JJ, Wigley FM, Shah AA, Corse AM, CasciolaRosen L, Hummers LK, et al. Association of fibrosing myopathy in systemic sclerosis and higher mortality. Arthritis Care Res (Hoboken) 2017;69:1764-70.

35. Prazma CM, Bel EH, Price RG, Bradford ES, Albers FC, Yancey SW. Oral corticosteroid dose changes and impact on peripheral blood eosinophil counts in patients with severe eosinophilic asthma: A post hoc analysis. Respir Res 2019;20:83.

36. Ortega H, Llanos JP, Lafeuille MH, Duh MS, Germain $\mathrm{G}$, Lejeune D, et al. Effects of systemic corticosteroids on blood eosinophil counts in asthma: Real-world data. J Asthma 2019;56:808-15. 